\title{
Design and implementation of magnetic field effect sensor for ISF process
}

DOI : 10.36909/jer.13469

\author{
Vilas H Gaidhane* and R Karthikeyan** \\ *Department of Electrical and Electronics Engineering and APPCAIR, Birla Institute of \\ Technology and Science Pilani, Dubai Campus, UAE \\ ** Department of Mechanical Engineering, Birla Institute of Technology \& Science Pilani, \\ Dubai Campus, UAE. \\ *Corresponding Author Email: vilasgd612@gmail.com
}

\begin{abstract}
In this paper, an efficient approach for sheet metal thickness measurement is proposed. It is based on the concept of magnetic field effect resonance commonly called as Hall effect. The combination of magnet and metal tools generates the DC voltage signal. The magnitude of the generated signal is inversely proportional to the distance between magnet and tool and hence the thickness of the sheet metal. The proposed idea is designed and implemented successfully using suitable hardware. Initially, the designed circuit is tested manually using the Hall-effect sensor and the sheet metal of different thicknesses. Further, the various experimentations are carried out on the complete CNC machine-based incremental sheet metal forming setup to examine the effectuality of the proposed design. Moreover, the evaluation of the manufactured ISF part has been carried out using the image processing technique. It is observed that the proposed approach can be used efficiently in the application such as the incremental sheet metal forming (ISF) process in real-time for thickness measurement.
\end{abstract}


Keywords: incremental sheet metal forming, hall effect, magnetic sensor, thickness measurement, image processing.

\section{INTRODUCTION}

Incremental sheet metal forming (ISF) is an important manufacturing process, ranging from small artificial human body parts to large aeroplane parts with complex shapes. The cost of the automotive process can vary from a few dollars to millions of dollars, depending on the complexity and the size of the parts (Shanbhag et al., 2018). ISF is a flexible sheet metal forming process in which the metal sheet part can form using a localized deformation. This process is simple and flexible because it does not require a specific tool or die. In this, the tool is moved along the predefined path with highly localized plastic deformation. A wide range of 3D parts can be manufactured using such a process.

ISF manufacturing is highly useful for small batches of products or customized parts. Moreover, in this process, dedicated toolsets are no longer required and hence reduce the time and production cost of the manufacturing of such complex products. It also provides higher formability and it can avoid the sheet necking problem due to its localized and incremental deformation nature (Malhotra et al., 2012). ISF is an improved technique of die-less sheet forming (DSF) techniques (Maidagan et al., 2007). In recent years, generally, two types of ISF processes are used for manufacturing sheet metal parts: single point incremental forming and double-point incremental forming. In the case of single point incremental forming, a blank holder and deforming tool are used for sheet formation (Kulkarni et al., 2004). In this, only one tool moves over the surface of the metal. The metal sheet clamped over a universal stationary blank holder and tool follow the predefined contour path. However, this approach results in less geometrical accuracy (Jeswiet et al., 2005). In the two-point sheet metal forming process, a blank holder, forming tool and support tool can be used. It has better accuracy as compared to the single point sheet metal forming process. 
Thickness is one of the important parameters and plays an important role in the ISF process.

It is well known that the thickness of the sheet can be calculated using the sine law given as (Jackson \& Allwood, 2009).

$$
T_{\text {final }}=T_{\text {initial }} \sin \left(90^{\circ}+\delta\right)
$$

where $T_{\text {initial }}$ is the initial thickness of the sheet and $\delta$ is the wall angle defines the angle between the un-deformed and deformed metal sheet.

The various experimentations are carried out based on the sine law on a truncated cone with various wall angles formed by the single point incremental forming (Young \& Jeswiet, 2004). However, it is observed that sheet thinning always does not follow the sine law and it can be used only when the strain is low. Further, the different variations and proposed geometrical model of the kinematics for the sheet metal forming process has been proposed in the literature (Bambach, 2010). However, these models are mostly depending on the material behaviour and parameter like friction.

In terms of the geometric accuracy, geometric error for ISF can only achieve around $\pm 2 \mathrm{~mm}$ due to the lack of support. The various approaches such as tool path compensation (Fiorentino et al., 2014), two moving forming tools (Emmens et al., 2010) and multistage forming (Liu et al., 2013) are proposed to improve the geometry accuracy. Cao et al. (2014) presented a new approach for thickness prediction in multi-pass incremental sheet metal forming. Kumar \& Gulati (2018) proposed an optimization technique to obtain an improved geometric thickness accuracy. These approaches are time-consuming and it has limited accuracy. Moreover, the thickness of the part is affected by the behaviour of the material and the friction due to the tools. Thus, geometrical inaccuracy, excessive thinning remains the challenge for ISF applications. To overcome these issues, an alternative approach based on the online measurement adaptive control technique can be used that avoid delay and fracture during the manufacturing process. In recent years, various researchers have carried out numerous 
research work and presented different methods to measure the online thickness of the ISF manufactured parts. Singh \& Agrawal (2017) developed a laboratory setup for a single point ISF process using a motion card to control the 3 servo motors for individual 3-axis control. Moreover, the strain over the entire length deformation has been computed. Dejardin et al. (2010) also state that the online monitoring of the thinning process of the sheet metal is an important task for successful manufacturing of the SPIF and optimization of the tool path. They proposed a new solution for thickness measurements based on the ultrasonic device integrated into a forming tool. However, in these methods, a propagation medium with specific viscosity is required. Another drawback of this method is that the setup can only be used in machines that can follow the normal vector defined by the tool centre point and contact points of the sheet.

In 2014, Paniti has proposed a cloud-based adaptive approach for ISF manufacturing and describes how the integrated approach provided flexible manufacturing to the industries. Moreover, a new adaptive tool path control algorithm at the process level has been introduced to overcome the fracturing issue due to the localized thinning. Further, an online thickness measurement approach based on the Hall-effect sensor integrated inside the tool has been proposed and applied successfully in the ISF application. However, the adjustment of the bearing and Hall-effect sensor in the tool remains a challenge for researchers (Paniti, 2014). Therefore, in this paper, a new online thickness measurement solution based on the Hall-effect sensor is proposed. The different ISF parts are manufactured using the proposed approach and online thickness measurement is carried out to validate the results. However, the evaluation of the sheet metal parts quality in the form of roughness is an important task at the end of the manufacturing process. Surface roughness is a quality that has a direct impact on performance and appearance. In recent years, various image processing-based surface roughness measurement methods have been proposed as an alternative to destructive and costly methods. It is observed in the literature that the image processing-based approaches outperform the 
direct contact methods (Gaidhane et al., 2018). Therefore, in this paper, an image processing technique has been used to evaluate the manufactured ISF parts.

\section{PROPOSED ONLINE THICKNESS MEASUREMENT TECHNIQUE}

In recent years, an integrated ultrasonic sensor has been used for thickness measurement. However, due to the cost and large size, this technique become less popular in the manufacturing industries. It is observed from the literature that the Hall-effect sensors can be an alternative for the measurement of thickness. In 2014, Paniti presented a flexible online system for thickness measurement which can be used in ISF manufacturing. They analyzed the possibilities of a Hall-effect sensor integrated with a flat surface tool. The experimentations using the Hall-effect sensor shows the good possibilities of thickness measurement. However, the flat tool shows inaccuracy which limits the uses. They proposed another new technique based on the bearing ball tool with a free rotation option. A Hall-effect sensor and magnet should be placed on either side of the sheet.

In literature (Hao \& Duncan, 2010) a detailed explanation of the ISF process has been discussed. They showed that the geometrical shape accuracy made by the ISF is lower than the stamping process. To overcome these issues, they proposed a closed-loop feedback control technique and adopt the linear forming process. Moreover, Polyblank et al. (2014) presented a systematic review on closed-loop control of manufacturing products in sheet metal forming. It is also observed that the tool path step depth $(d t)$ is an important parameter in the ISF process. The lower step depth increases the accuracy, on other hand increases the processing time also. This leads to inefficient product manufacturing in the industry. 


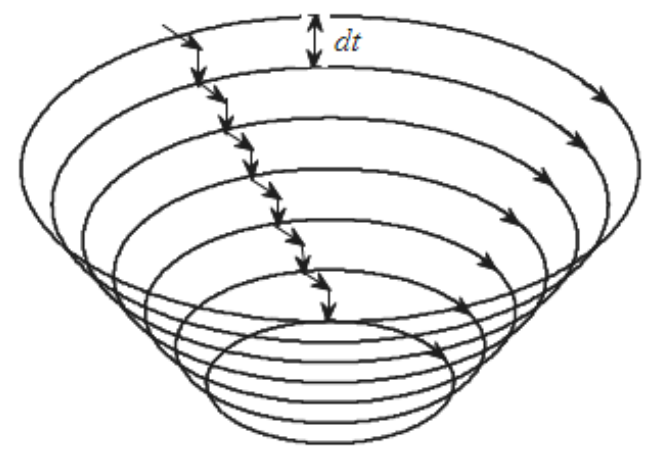

Fig. 1 Illustration of tool path and step depth

A possible solution may be an online thickness measurement with as simple adaptive control techniques which changes the step depth to the desired value. Fig. 1 shows the trajectory of the tool path with step depth $(d t)$. In order to measure the metal sheet thickness with a Hall-effect sensor, the surrounding setup should make up of non-ferromagnetic material. Recently, a technique has been presented to measure thickness using a Hall sensor and feedback control system. However, they have used a flat magnet which results in more friction between the magnet and sheet surface. To overcome the friction issue, the machine oil is used as a lubricant in the process.

In this work, an attempt has been made and a new approach has been proposed to measure thickness online using the concept of hall-effect sensor and magnet. A magnet ball is used in the setup which results in minimum sheet-metal and magnet ball contact area. Thus, the presented approach avoids the use of machine oil lubricant and results in a simple and efficient metal sheet thickness measurement system.

\section{WORKING PRINCIPLE AND CALIBRATION OF HALL EFFECT SENSOR}

It is well known that the magnetic field configuration can provide the linear flux density as a function of mechanical displacement and it is depending on the size of the magnet. The basic principle of Hall-effect and magnetic flux density is inversely proportional sheet thickness. The Hall Effect sensor is to be placed on one side inside the tool and a magnet on the other 
side of the sheet. The magnet follows the sensor along with the programmed tool path. The setup is adjusted according to the thickness of the metal sheet.

A linear output Hall Effect sensor (Allegro A1324) IC has been used in the experiments. Moreover, a neodymium magnet of $10 \mathrm{~mm}$ diameter provides a high magnetic field. Initially, the proposed system is calibrated offline assembling the components like sensor and magnetic with screw gauge to measure the optimum thickness of the metal sheet.

The magnetic flux density varies monotonically with respect to the displacement. However, the resultant voltage generated by the Hall Effect sensor is nonlinear. Therefore, it is required to calibrate properly before actual use in real-time application in the system. The complete assembly for the offline calibration of the Hall Effect sensor with a micrometer screw gauge is prepared on the breadboard. The calibration has been carried out in 10 to 15 series of reading using the Arduino Uno R3 microcontroller. In This, it is necessary to maintain the appropriate distance between the magnet and sensor. The second-order polynomial equation gives the relation between the voltage and the distance which can be represented as

$$
y=0.0025 x^{2}-0.0323 x+2.854
$$

Fig. 2 shows the calibration curve with the distance between the magnet and sensor (considered as a thickness in $\mathrm{mm}$ ) and the output voltage of the sensor.

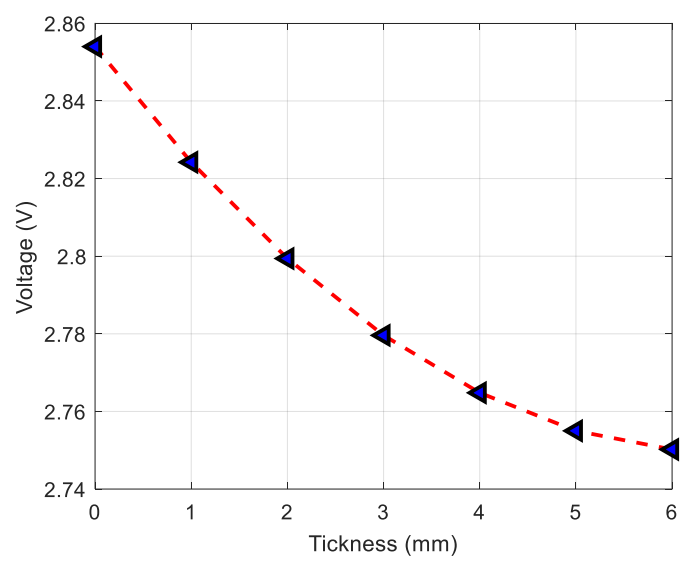

Fig. 2 Calibration curves with different magnet-sensor distances 
In traditional machining operations such as turning, milling, grinding and drilling are the common operations in the manufacturing industry (Zhang et al., 2016). While the metal sheet is machined for the predefined shape, the forming tool design plays an important role. In this work, a circular shaped tool is prepared and used for the various experimentations. The Halleffect sensor is also fitted inside the notch which is formed on the surface of the tool. Fig. 3 shows the Hall-effect sensor integrated tool used for the sheet thickness measurement applications.

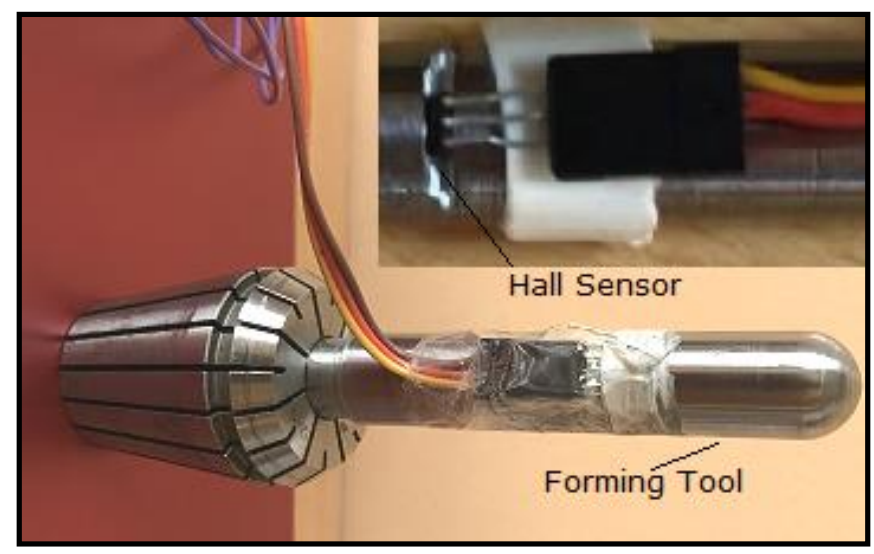

Fig. 3 Hall-effect sensor integrated forming tool

\section{EXPERIMENTAL RESULTS AND ANALYSIS}

In this research work, an attempt has been made to build a system to measure the thickness of metal sheets online in the ISF process. In recent years, few researchers have presented the thickness measurement techniques that involve the ultrasonic sensor (Dejardin et al., 2010). However, this system has some challenges such as big size, installation space, and the requirement of lubricants, which make it impractical. Therefore, it is required to ensure that the designed system is small in size and nondestructive.

The experimental set involves the integrated tool and 3-axis $\mathrm{CNC}$ vertical milling machine (Electrical Specifications: Input Voltage $-415 \pm 5 \% \mathrm{~V}$, Frequency $50 \pm 1 \% \mathrm{~Hz}$, Input power - 20kW, Mechanical Specifications: BT 30/40 Spindle with $8000 \mathrm{rpm}$, Tool weight $-3 \mathrm{~kg}$ ). 
A cylindrical steel made tool with a hemisphere head of diameter $14 \mathrm{~mm}$ is used as forming tool. A small notch approximately $25 \mathrm{~mm}$ away from the head of the tool is used to fit the Hall sensor. From testing of the setup, it is observed that the minimum distance between the magnet ball and sensor need to be at least $25 \mathrm{~mm}$ to observe the changes in the voltage values.

An Aluminium sheet $250 \times 250 \mathrm{~mm}$ with a thickness of $1 \mathrm{~mm}$ has been fixed onto a fixture that is loaded onto the $\mathrm{CNC}$ machine setup. The part to be designed in these experiments is a truncated cone, with a diameter of $100 \mathrm{~mm}$ and a depth of $60 \mathrm{~mm}$. Moreover, the wall angle of the part is $60^{\circ}$. The step-down value of the tool is $0.6 \mathrm{~mm}$. The setup for the detection of sheet thickness consists of a linear output Hall effect sensor (Allegro A1324) and a neodymium magnet of $10 \mathrm{~mm}$ diameter. The complete system for online thickness measurement is shown in Fig. 4.

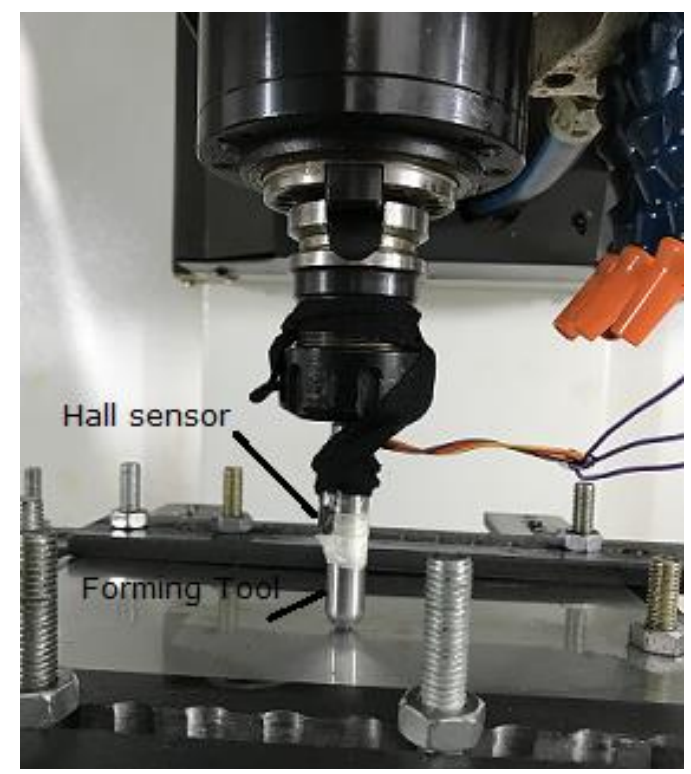

Fig. 4 Setup for the experimentation

The various SIEF experiments with hall effect sensors are carried out with three different $0.4 \mathrm{~mm}, 0.5 \mathrm{~mm}$ and $0.7 \mathrm{~mm}$ thick aluminium sheets. The sheet forming process and the position of the magnetic sensor following the tool position is shown in Fig. 5 (a) and (b), respectively. 


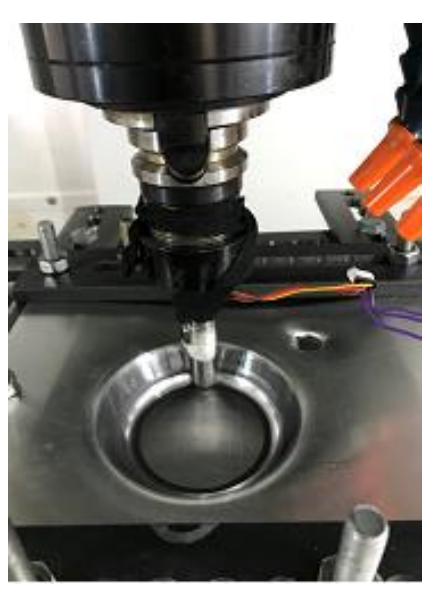

(a)

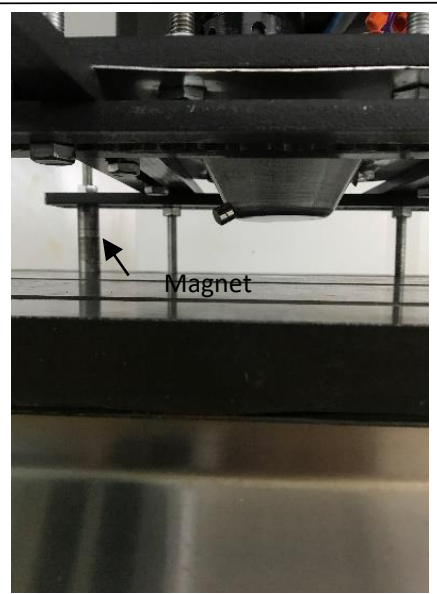

(b)

Fig. 5 Setup with metal sheet and Hall effect sensor: (a) Sheet forming in process, (b) Magnet following the tool.

Initially, the ISF has been carried out with $0.4 \mathrm{~mm}, 0.5 \mathrm{~mm}$ and $0.7 \mathrm{~mm}$ aluminium sheets and obtain the various values voltages for sheet thickness at different points. However, it is observed that the output voltage level of the magnetic sensor varies continuously. Therefore, the calibration of the proposed designed Hall effect sensor has been carried out for all these three metal sheets. The voltage levels at a different distance from the metal sheet surface for all these three sheets are shown in Fig. 6.

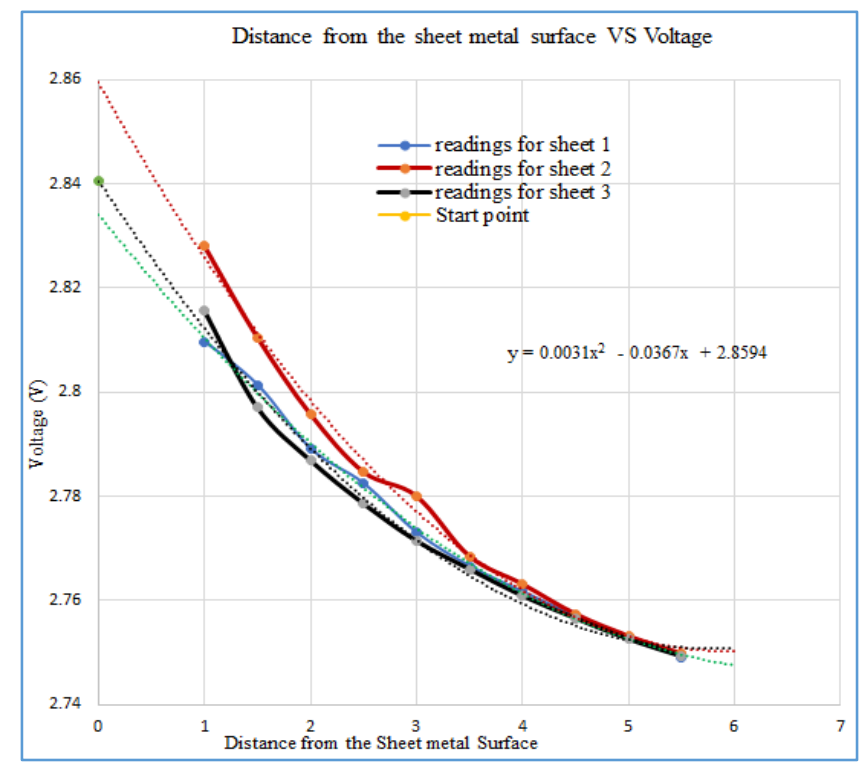

Fig. 6 Voltage obtained at a different distance $(\mathrm{mm})$ from the sheet metal surface for sheet metal 1 , sheet metal 2 and, Sheet metal 3, respectively. 
It is observed from Fig. 6 that the sensor follows the nonlinear relation between the distance between the magnet and sensor and the respective voltage for all three metal sheets. The second-order polynomial gives the relation between voltage and distance between the magnet and sensor which can be represented as

$$
y=0.0031 x^{2}+0.036 x+2.8594
$$

The raw data obtained from the sensor is shown in Fig. 7. The noise may be present in the acquired signal due to vibration of the machine, movement of the magnet and also the inherent noise of the Hall effect sensor. From Fig. 7(a)-(c), it is observed that the voltage level is different at some points which show the ununiform thickness of the metal sheet.

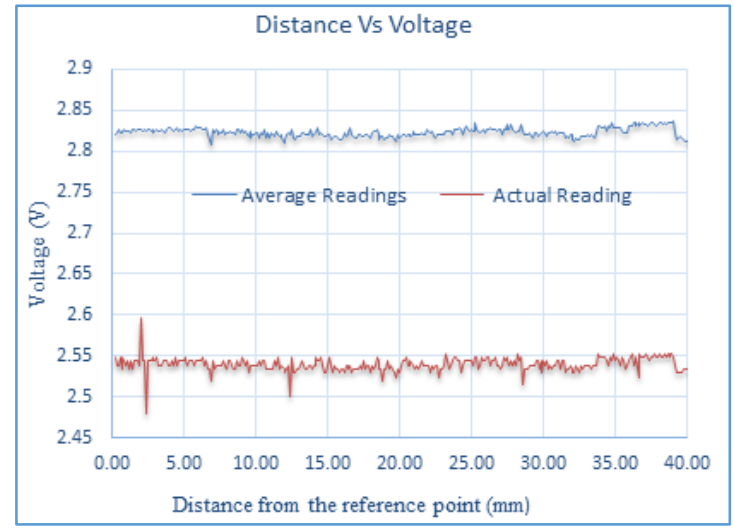

(a)

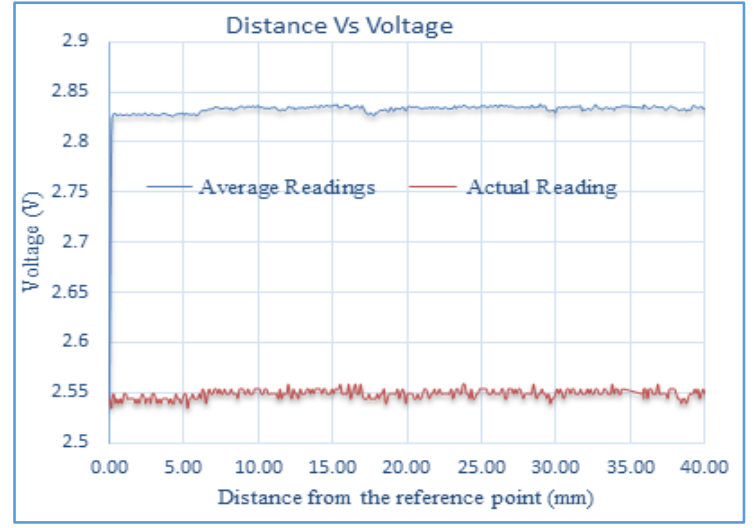

(b)

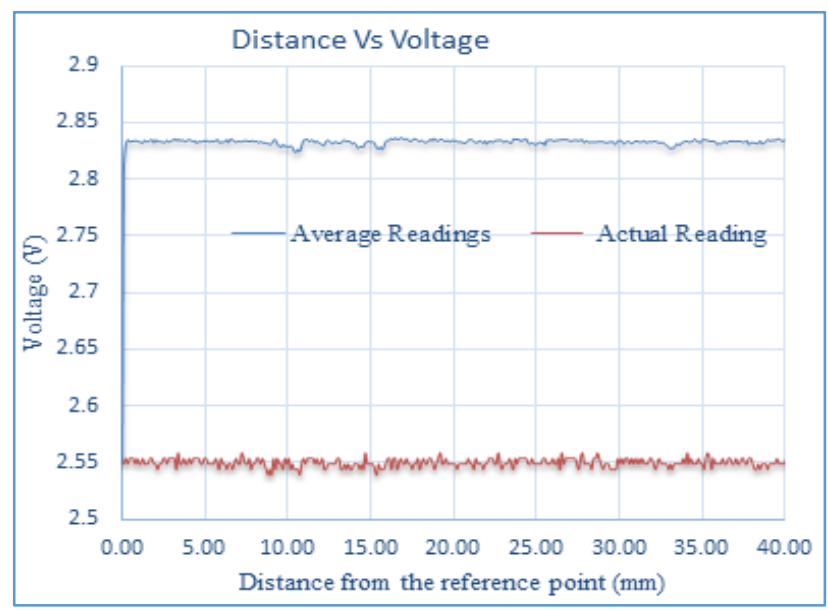

(c)

Fig. 7 Voltage obtained at different points located at the distance $(\mathrm{mm})$ from the reference point: (a) Sheet metal 1, Sheet metal 2 and, (c) Sheet metal 3. 
From Fig. 7, it is observed that the variation in the voltage value is nonlinear at the different instants of observation. This can be caused due to the presence of errors from various sources. While performing the experiments on three aluminium metal sheets, the sources of error are identified and their values are also quantified. The first source of error encountered during the experimentations is the inherent noise of the sensor. Even though the magnetic field around the sensor is zero, fluctuations are observed in the output voltage. The value of the error is calculated by finding the standard deviation of the readings. The second source of error is due to the non-uniform surface of the sheet. Even though precautions are taken to ensure uniform thickness over the entire sheet slight errors can arise due to various reasons. The third source of error is the movement of the magnet. Since this is a single point forming process, the magnet may be stuck under the sheet. Moreover, due to friction between the magnet and the sheet, the movement of the magnet may not be uniform and hence a non-uniform magnetic field can be experienced by the Hall effect sensor.

Table 1 Sources of Error in the system

\begin{tabular}{|l|c|}
\hline Sources of Error & Error (Volt) \\
\hline Noise in the sensor & $9 \times 10^{-4}$ \\
\hline Error due to Non-uniform surface & 0.0155 \\
\hline Error due to movement of a magnet & 0.0148 \\
\hline
\end{tabular}

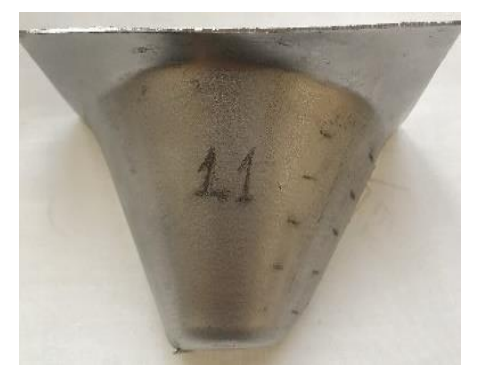

ISF part 1

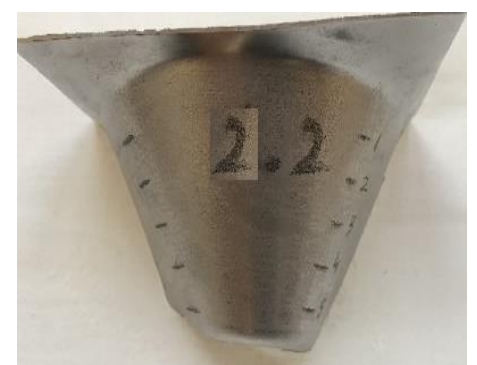

ISF part 2

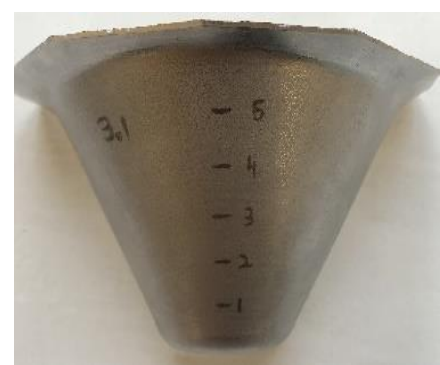

ISF part 3

Fig. 8 Cone structure formed by proposed ISF process. 
Further, the ISF process has been carried out on various aluminium sheet metal plates and the various cone structure are made using the proposed method as shown in Fig. 8. The uniformity of the thickness is measured by the Hall effect sensor and maintained by the closedloop control system.

In recent years, various metal sheet surface roughness evaluation techniques have been proposed as an alternative to the destructive and costly method. Image processing is one of the low cost and effective approaches which can be used to evaluate the surface roughness of the ISF part surface (Gaidhane et al., 2018, Rajevenceltha \& Gaidhane, 2021). In this experiment, various sheet metal parts have been considered as shown in Fig. 9.

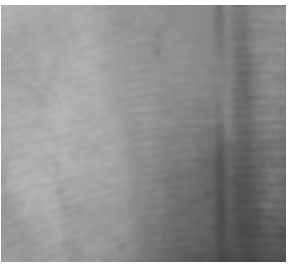

(a)

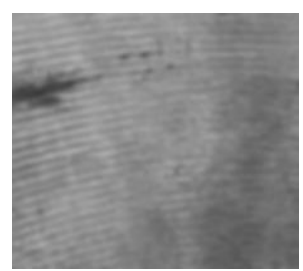

(c)

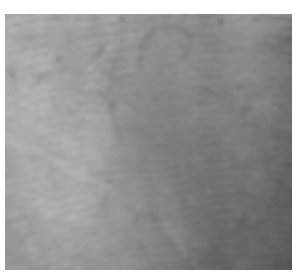

(e)

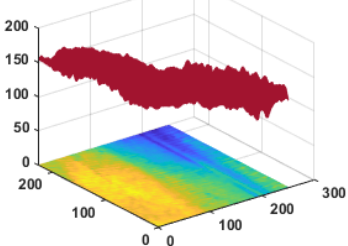

$(\mathrm{g})$

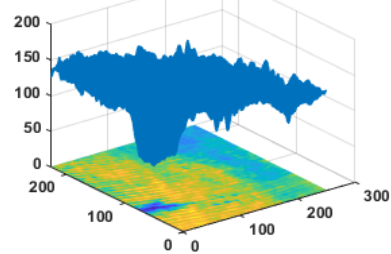

(i)

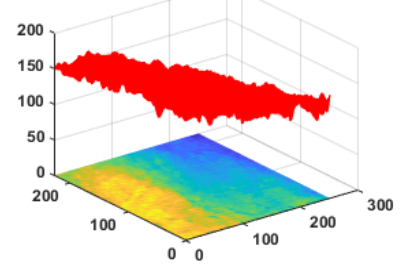

(k)

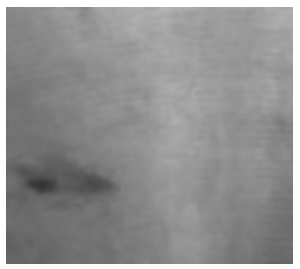

(b)

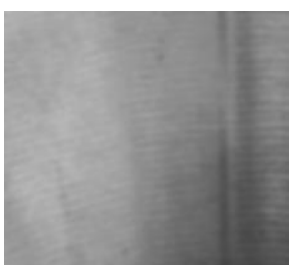

(d)

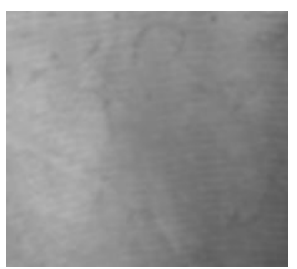

(f)

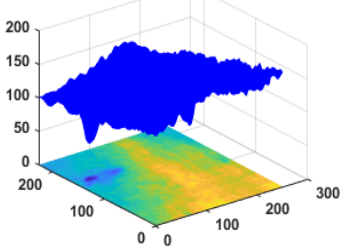

(h)

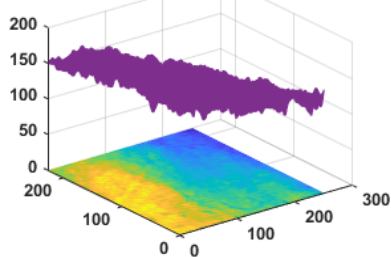

(j)

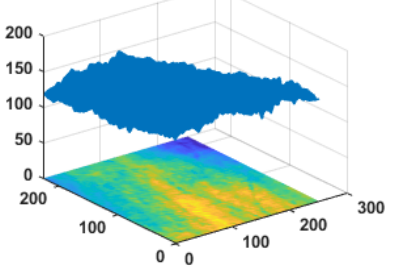

(1)

Fig. 9 Sheet metal surface and roughness; (a)-(f) metal plate area, (g)-(l) graphical representation of roughness of sheets metal surface. 
The contrast variation may be present in the acquired images. Therefore, preprocessing is carried out to remove such undesirable effects from the images. In this experiment, a Gaussian filter is used to remove the contrast effect present in the images. The various experimentations are carried out using the Gaussian filter and image processing tool to study the roughness of the ISF sheet metal parts. Fig. 9(a)-(f) shows the surface area of six different ISF parts. Moreover, Fig. 9(g)-(l) shows the graphical representation of the roughness of the surface of sheet metals, respectively. It is observed that the roughness of the sheet metal shown in Fig. 9(a) is less and hence the spikes and variations are very less in respective graphical representation as shown in Fig.9(g). However, Fig. 9(h) and (i) shows more spikes, as well as varied as the sheet metal surface, is rough. Thus, the image processing technique can be used efficiently to evaluate the surface of manufactured parts using the ISF process.

\section{CONCLUSION}

In this paper, a simple and efficient method for incremental sheet metal forming is proposed. The presented method is based on the concept of the Hall effect phenomenon. The magnetic effect sensor is designed and implemented successfully to measure the thickness of the ISF parts at various points. Moreover, the image processing technique is also used to evaluate the roughness of the formed parts. It is observed that the proposed magnetic field effect sensor outperforms the costly, destructive and surface contacts methods. Moreover, it is robust and performs better in the presence of noise and variations. This approach can be applied in various defect detection as well as ISF applications.

The proposed design can be used in various applications such as incremental sheet metal parts manufacturing. In biomedical applications, the manufacturing of the artificial metal sheet based part requires high accuracy and dimensions. Moreover, most of the aeroplane parts need accurate dimensions and a uniform surface. In such applications, the presented approach can be used effectively to manufacture sheet metal parts. In future, the Hall effect sensor-based approach can be integrated with intelligent control with image processing techniques. 


\section{REFERENCES}

Shanbhag, V.V., Rolfe, B.F., Arunachalam, N. \& Pereira, M.P. 2018. Investigating galling wear behavior in sheet metal stamping using acoustic emissions. Wear 414-415: 31-42.

Malhotra, R., Xue, L., Belytschko, T. \& Cao, J. 2012. Mechanics of fracture in single point incremental forming. Journal of Materials Processing Technology. 212(7): 1573-90.

Maidagan, E., Zettler, J., Bambach, M., Rodríguez, P.P. \& Hirt, G. 2007. A new incremental sheet forming process based on a flexible supporting die system. Key Engineering Materials 344: 607-14.

Kulkarni, D.M., Prakash, R., Talan, P. \& Kumar, A.N. 2004. The effect of specimen thickness on the experimental and finite element characterization of CTOD in extra deep drawn steel sheets. Sādhanā 29(4): 365-80.

Jeswiet, J., Micari, F., Hirt, G., Bramley, A., Duflou, J. \& Allwood, J. 2005. Asymmetric single point incremental forming of sheet metal. CIRP Annals 54(2): 88-114.

Jackson, K. \& Allwood, J. 2009. The mechanics of incremental sheet forming. Journal of Material Processing Technology 209(3): 1158-74

Young, D. \& Jeswiet, J. 2004. Wall thickness variations in single-point incremental forming Journal of Engineering Manufacturing, Part B. 218: 1453-59.

Bambach, M. 2010. A geometrical model of the kinematics of incremental sheet forming for the prediction of membrane strains and sheet thickness. Journal of Material Processing Technology 210(12): 1562-73.

Fiorentino, A., Feriti, G.C., Ceretti, E., Giardini, C., Bort, C.M.G. \& Bosetti, P. 2014. Development of tool path correction algorithm in incremental sheet forming. Key Engineering Materials 622: 382-89. 
Emmens, W.C., Sebastiani, G. \& Van den Boogaard, A.H. 2010. The technology of incremental sheet forming - a brief review of the history. Journal of Material Processing Technology 210(8): 981-97.

Liu, Z., Li, Y. \& Meehan, P.A. 2013. Vertical wall formation and material flow control for incremental sheet forming by revisiting multistage deformation path strategies. Material and Manufacturing Processes 28(5): 562-71.

Cao, T., Lu, B., Xu, D., Zhang, H., Chen, J., Long, H. \& Cao, J. 2014. An efficient method for thickness prediction in multi-pass incremental sheet forming. International Journal of Advanced Manufacturing Technology. 77(1-4): 469-83.

Kumar, A. \& Gulati, V. 2018. Experimental investigations and optimization of forming force in incremental sheet forming. Sādhanā 43(10): 159.

Singh, A. \& Agrawal, A. 2017. Experimental force modeling for deformation machining stretching mode for aluminium alloys. Sādhanā. 42(2): 271-80.

Dejardin, S., Gelin, J.C. \& Thibaud, S. 2010. On-line thickness measurement in incremental sheet forming process. Steel Research International 81(9): 938-41.

Paniti, I. 2014. Adaptation of incremental sheet forming into cloud manufacturing. CIRP Journal of Manufacturing Science and Technology 7(3): 185-90.

Gaidhane, V.H., Hote, Y.V, \& Singh, V. 2018. An efficient similarity measure approach for PCB surface defect detection. Pattern Analysis and Applications 27: 277-89.

Gaidhane, V.H. \& Hote, Y.V. 2018. An efficient edge extraction approach for flame image analysis. Pattern Analysis and Applications 21(4), 1139-50.

Paniti, I. \& Paróczi, A. 2011. Design and modeling of integrated Hall-effect sensor-based online thickness measurement device for incremental sheet forming processes. In proceedings of 2011 IEEE/ASME International Conference on Advanced Intelligent Mechatronics (AIM) Budapest, Hungary, pp. 297-302. 
Hao, W. \& Duncan, S. 2011. Optimization of tool trajectory for incremental sheet forming using closed-loop control. Proceedings of 2011 IEEE International Conference on Automation Science and Engineering, Trieste, Italy, pp. 779-84.

Polyblank, J.A., Allwood, J.M., \& Duncan, S.R. 2014. Closed-loop control of product properties in metal forming: A review and prospectus. Journal of Material Processing Technology 214(11): 2333-48.

Rajevenceltha, J. \& Gaidhane, V.H. 2021. An efficient approach for no-reference image quality assessment based on statistical texture and structural features. Engineering Science and Technology, An International Journal, https://doi.org/10.1016/j.jestch.2021.07.002.

Rajevenceltha, J \& Gaidhane, V.H. 2021. A novel approach for image focus measure. Signal, Image and Video Processing 15(3): 547- 55.

Zhang, C., Yao, X., Zhang, J. \& Jin, H. 2016. Tool condition monitoring and remaining useful life prognostic based on a wireless sensor in dry milling operations. Sensors 16(6): 795. 